\title{
Developments in Test Facility and Data Networking for the Altitude Test Stand at the John C. Stennis Space Center, MS - A General Overview
}

\author{
Phillip W. Hebert' Sr. \\ Engineering \& Sciences Directorate, NASA John C. Stennis Space Center, Stennis Space Center, MS 39529, USA
}

\begin{abstract}
May 2007, NASA's Constellation Program selected John C Stennis Space Center (SSC) near Waveland Mississippi as the site to construct an altitude test facility for the developmental and qualification testing of the Ares1 upper stage (US) engine. Test requirements born out of the Ares1 US propulsion system design necessitate exceptional Data Acquisition System (DAS) design solutions that support facility and propellant systems conditioning, test operations control and test data analysis. This paper reviews the new A3 Altitude Test Facility's DAS design requirements for real-time deterministic digital data, DAS technology enhancements, system trades, technology validation activities, and the current status of this system's new architecture. Also to be discussed will be current network technologies to improve data transfer.
\end{abstract}

\section{Introduction}

The A-3 test facility is a test facility designed to perform altitude testing of the Ares1 upper stage (US) engine. The A-3 test facility is a challenge for all engineering disciplines at Stennis Space Center, including the electrical systems designers. In order to obtain and maintain vacuum conditions for the US engine, a two-stage diffuser with a Chemical Steam Generator (CSG) system is employed. The two-stage diffuser contains a vertical section, referred to as the "first stage" and a horizontal section, referred to as the "second stage". The height required for the vertical section results in the exit plane of the US engine nozzle and the test level of the stand being several hundred feet above grade in order to function properly. A vacuum chamber, otherwise known as the test cell, is used to seal and maintain vacuum conditions for US engine testing. Propellant run tanks which provide fuel and oxygen to the US engine are also required for US engine testing. These tanks are mounted exterior to the vacuum chamber and above the US engine propellant inlet piping to provide proper propellant flow. An open steel frame structure provides structural support of the vacuum chamber, diffuser, the propellant run tanks, and housing for a tool crib and electronics required to interface with the US engine. A pictorial model of the A-3 test facility is shown in Figure 1.

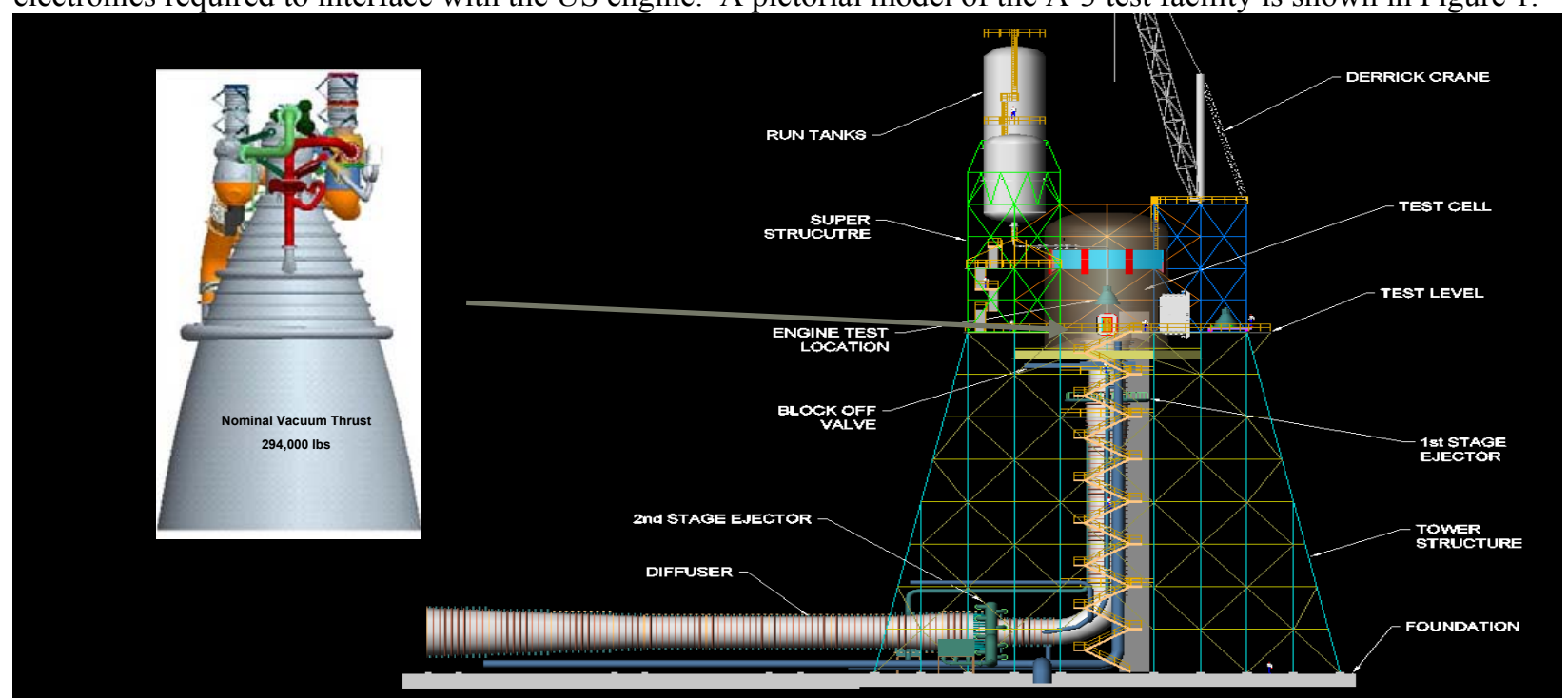

Figure 1 : Pictorial model of the A-3 test facility

1

American Institute of Aeronautics and Astronautics 
For the A-3 test facility, Measurement, Monitor, and Control Systems (MM\&CS) are required to perform facility operations and acquire data for US engine certification, particularly the engine thrust level required for achieving mission requirements. Design of the MM\&CS must consider all aspects during the design process, including some unusual design requirements for SSC. The electrical systems design consists of penetration of the test cell while implementing state of the art technical systems to provide safe control and monitoring of the US Engine. In order to accomplish these tasks, designers must be cognizant of the ever emerging technologies and possess the ability to implement these technologies in order to meet program requirements.

The A-3 test facility MM\&CS encompasses High and Low Speed Data Acquisition Systems (HS \& LSDAS), controls systems to control facility and steam generator operations, High and Low Speed Video systems, ancillary systems, Hydrogen Fire and Gas detection systems, and an overall test facility network system which interconnects all of these sub-systems. Figure 2 depicts these systems, their interactions and their connectivity. Due to the nature of the equipment employed in the MM\&CS, environmental conditioning is required to house the core components of the MM\&CS. The A-3 test facility design team determined that operational efficiency is obtained by housing this type of equipment in two separate environmental enclosures, referred to as Signal Conditioning Buildings (SCBs). One is located on the test stand at the test level and the other is located on the ground level to interface with the CSGs, which are also on the ground. For personnel safety, the main operator control functions for the MM\&CS will be housed in the Test Control Center (TCC), which is located a safe distance from the test stand and the SCBs. Therefore, the MM\&CS connections between the SCBs and the TCC use a fiber optic backbone for communication.

This paper provides a general description of each major MM\&CS subsystem and describes how each of these systems are using updated data networking technology to provide accurate and reliable operations for the A-3 test facility.

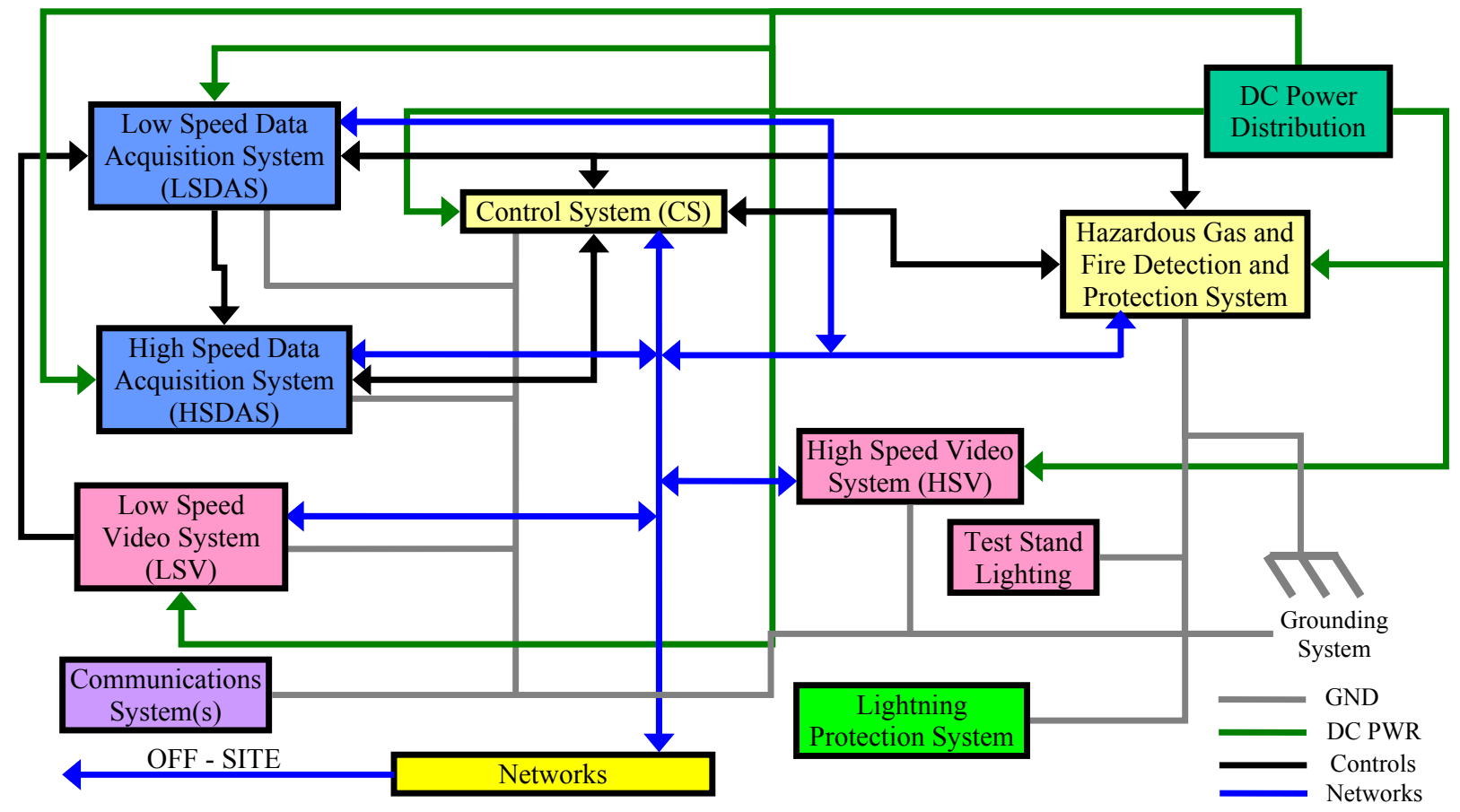

Figure 2 : A-3 test facility Electrical Systems (MMC) and Interactions ${ }^{1}$

\section{HSDAS}

For proper verification of the US engine design, instruments which measure engine performance, vibration, and fatigue are required. Many of these instruments are dynamic in nature, requiring High Speed Data Acquisition (HSDAS) systems providing high sample rate recording to acquire data for future analysis in the frequency domain. The A-3 HSDAS consists of signal conditioning, digitization, and remote recording of dynamic instrumentation. For the A-3 test facility, approximately 200 HSDAS channels are required to be sampled at rates higher than 102,400 samples per second (sps). In order to provide such a channel count with a high sample rate with remote 
recording, the use of the latest available network technology is required, therefore, the design of the A-3 test facility HSDAS utilizes a gigabit ethernet network backbone for transfer of data between the SCBs and the TCC.

The block diagram in Figure 3 represents the proposed architecture to support the channel requirements of the A3 test facility testing of the US engine. The SCB depicted in this diagram is the one located on the test stand at the test level. The redundant recording and control PC is located in the TCC for personnel safety and protection of test data. Since the TCC is not immediately adjacent to the SCB where the acquisition equipment resides, data is transmitted over a long distance single-mode fiber optic communications link using a gigabit ethernet switch.

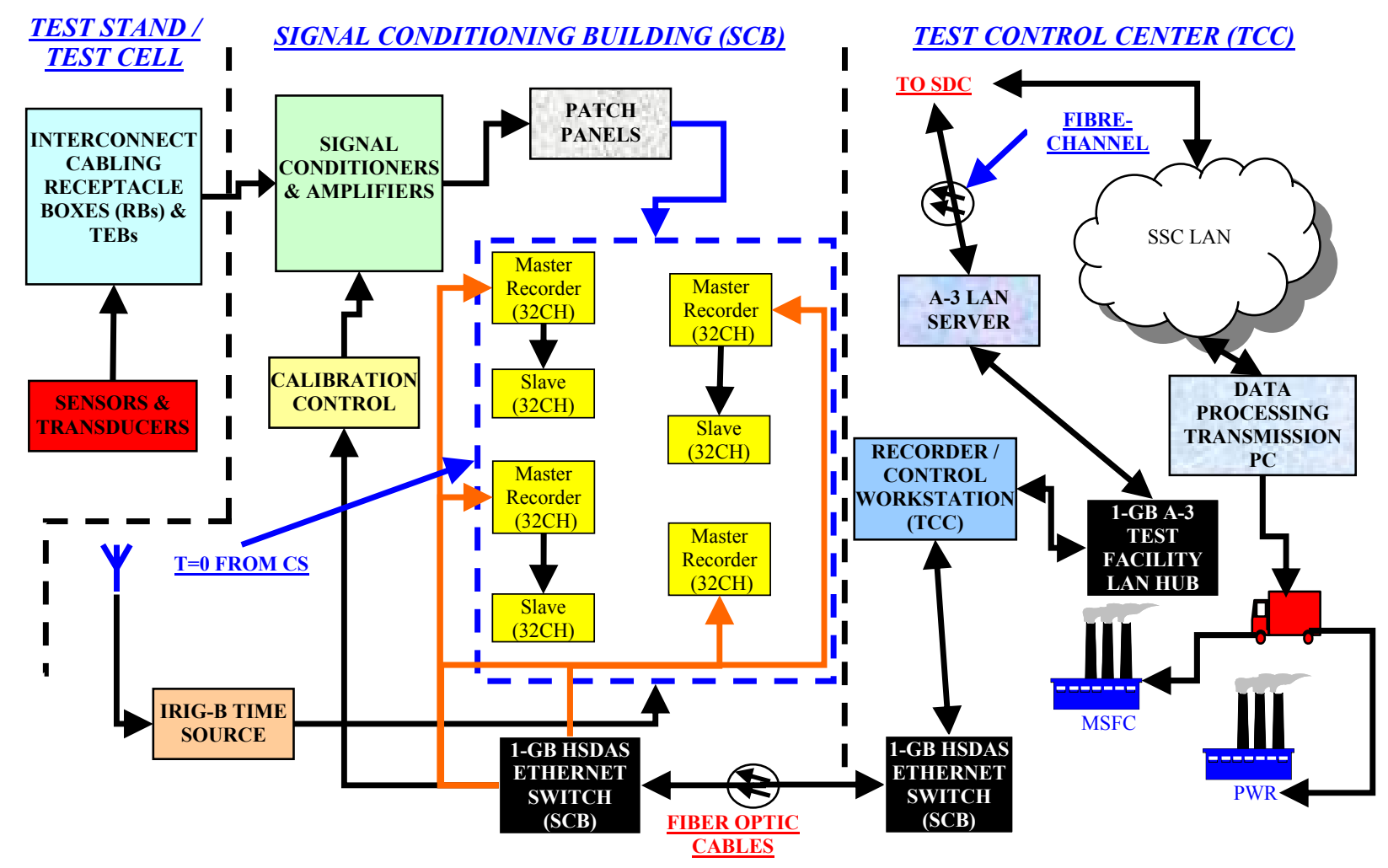

Figure 3 : A-3 HSDAS BLOCK DIAGRAM ${ }^{2}$

\section{A. Signal Conditioning}

In order to properly interface the digitizing and recording system with the instruments used to acquire dynamic measurement information, high speed signal conditioning is required. Signal conditioning provides the measurement device with the proper excitation, gain, filtering, and bridge completion if necessary. The signal conditioner then supplies an analog output to the digitizer and recording system with a signal in the proper voltage and frequency range for recording the data, which can be later retrieved and transformed into a signal that can be analyzed in the frequency domain. Typical types of devices with which the signal conditioning interfaces are accelerometers which measure vibration, dynamic pressure measurements which measure changing pressure, and strain gages which measure fatigue in test article components. Accelerometers can output either a charge per unit of vibration (commonly known as g's or a relation of acceleration due to gravity) which are known as charge mode devices, or a low impedance voltage mode which are known as IEPE or Integrated Electronics PiezoElectric devices. Dynamic pressure sensors typically output a voltage, using IEPE technology. Strain gages are typically configured as one leg of a Wheatstone bridge, thus requiring bridge completion resistors, which often times are resident on the signal conditioners. Wheatstone bridges require an excitation voltage be supplied by the signal conditioner and outputs a low-level signal, thus requiring amplification to properly provide a voltage signal to the digitizing and recording system.

For the A-3 test facility, signal conditioning which takes advantage of remote command and monitoring capabilities is being procured. The ability to use remote command and monitoring capabilities allows for the HSDAS to implement automated calibration capabilities which have historically been difficult to implement due to the lack of remote command and monitoring capabilities. The ability to implement automated calibration 
capabilities on a HSDAS can greatly reduce the time required for pre-test HSDAS validation, which ultimately results in lower costs to maintain and operate the system. A specification has been written and released to industry for HSDAS signal conditioning which takes advantage of current technology capabilities for use at the A-3 test facility as well as other test stands at SSC.

\section{B. Digitizing and Recording Function}

The digitizing and recording functions convert analog signals from the signal conditioners to digital data which is then recorded to a hard drive by the recording system. The digitizer and recorders are configured in a masterslave configuration with one master operating one slave unit, with each unit containing thirty-two channels of analog data and sixteen channels of discrete data. These units reside in the SCB on the test level of the test stand. The master unit is connected to the gigabit ethernet switch located in the SCB.

The high-speed recording system is controlled through the recorder / control workstation located in the TCC, which is connected on the other end of the gigabit ethernet switch located in the TCC. From this control PC, test personnel are able to initiate and terminate test recording. Recorded test data can also be replayed if desired.

\section{Data Processing}

Data Processing is performed in the master / slave units located in the SCB. The initiation of this process is performed using the recorder / control workstation in the TCC. Data is processed to produce verifiable propulsion test data by following a strict process for receiving, processing, delivering, and archiving test data. Recorded HSDAS data is converted to scaled (Engineering Unit) Fast Fourier Transforms (FFTs) for frequency analysis, and then transmitted to analysts who review the data to verify engine performance. Raw data is provided to the customer on a hard drive for archiving.

\section{LSDAS}

The Low Speed Data Acquisition System (LSDAS) performs a similar function to the HSDAS but records data from instrumentation that is more static in nature, thus not requiring equipment to record data at a high sample rate. For the A-3 test facility, approximately 800 LSDAS channels are required to be sampled at rates of approximately 250 samples per second (sps). The proposed architecture for the A-3 test facility LSDAS is depicted in Figure 4. The components are similar in function to the HSDAS, with signal conditioning, digitization, and recording equipment comprising the core functionality of the LSDAS. Peripheral components of the LSDAS include Receptacle Boxes (RBs) and cabling to connect the sensors to the various LSDAS components, such as sensors, $\mathrm{RBs}$, and signal conditioners. Receptacle Boxes (RBs), provide connection points for data system transducers and are located at various locations throughout the test stand. At the A-3 test facility some of these receptacle boxes are housed within the test cell, therefore, special considerations must be factored into the design to accommodate vacuum conditions and penetration methods of the signals through the test cell. Signal conditioning performs a similar function to the signal conditioning modules for the HSDAS, with the exception that additional types of instruments are accommodated. For the A-3 test facility, the digitizing function is intended to be provided on the same module as the signal conditioning, although the final architecture for this function has yet to be determined. These components will reside in both of the SCBs located on or near the test stand. Discrete events are also acquired using this function. The calibration control function allows remote control of the signal conditioning units which allows for the LSDAS to perform automated calibration functions. The recording and control computers provide control and recording capability for the LSDAS components, such as the signal conditioners and digitizers. Network switches and cabling provide interconnect capability for the LSDAS between the SCBs at the test stand and the TCC. The large channel count requires this network capability to be single-mode fiber optic connections of gigabit ethernet capability. Remote displays allow any user connected to the LSDAS network to view real-time data on a computer screen. The DAS real-time transmission server streams live test data outside of the Test Facility Local Area Network (LAN) to remote viewers. 


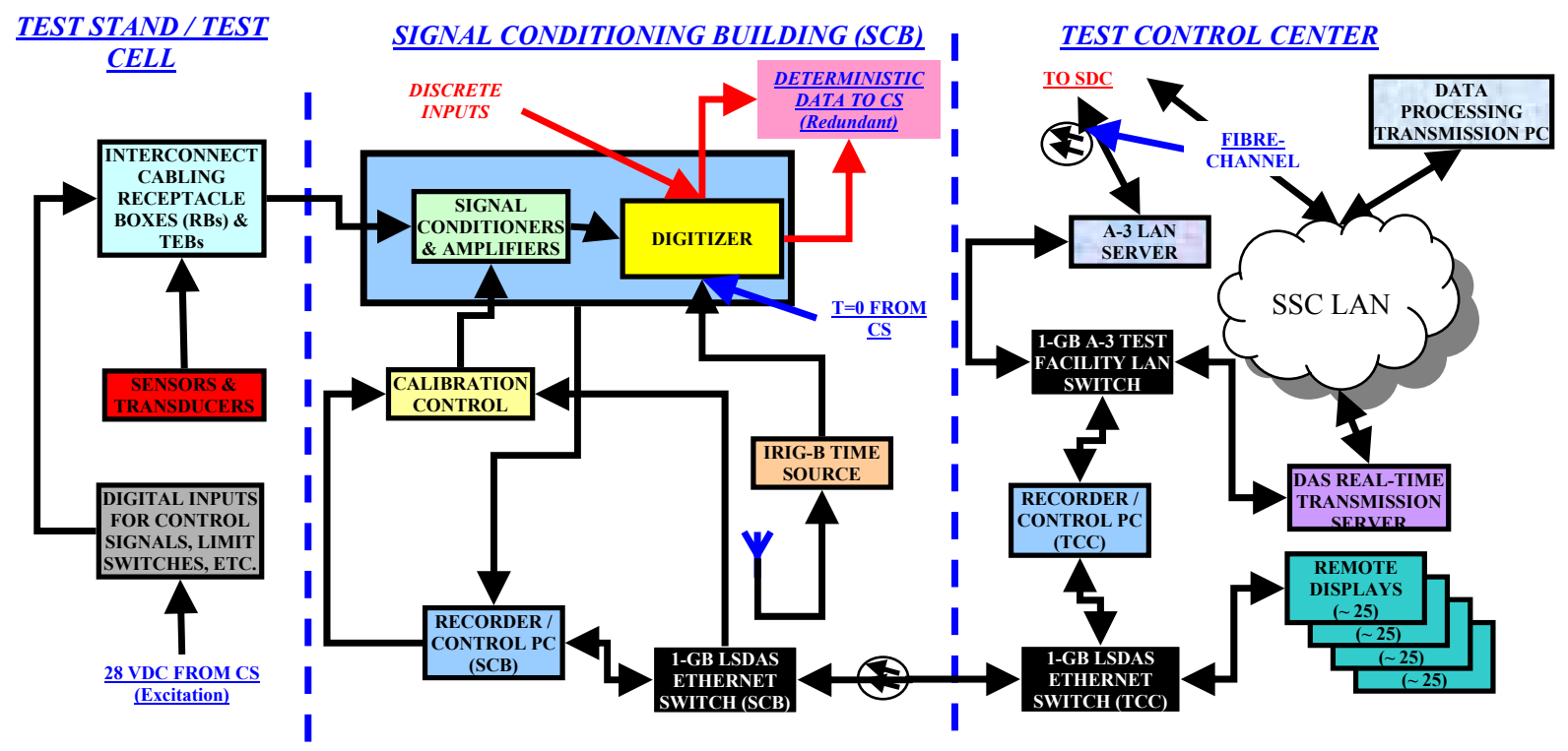

Figure 4 : A-3 test facility Low Speed Data Acquisition System (LSDAS)

\section{A. Signal Conditioning}

Signal conditioning equipment serves a similar function within the LSDAS as described previously, with the exception that the instruments are static in nature, whereas HSDAS measurements are more dynamic in nature. Signal conditioning for the A-3 LSDAS interfaces with such devices as pressure transducers, requiring bridge completion, thermocouples and Resistive Temperature Devices (RTDs) which measure temperature within the process flow. Other types of interfaces provided are voltage inputs for feedback signals from Variable Position Valves (VPVs), current inputs, which accept inputs typically in the 4-20 mill-ampere range, and digital inputs, which accept discrete event inputs. The system must accommodate an IRIG-B time code input which is used for correlation with other data systems such as the HSDAS and the video monitoring system. The signal conditioners for the A-3 test facility will be programmable similar to the HSDAS signal conditioning, which enables automated calibrations.

\section{B. Digitizing and Recording Function}

At SSC, the digitizer has historically been a separate piece of hardware from the signal conditioning hardware requiring them to be wired together. This separate implementation allows flexibility to route signal outputs from signal conditioners to multiple destinations. When engineers surveyed available capability in a market survey to determine the current capability of the industry, it was learned that a many vendors currently provide this function integral to the signal conditioning units ${ }^{3}$. This implementation significantly reduces the costs and space required for an LSDAS. Therefore, the LSDAS digitizer function for the A-3 test facility is intended to be integral to the signal conditioning. With this architecture in mind, NASA has written a specification which requests the LSDAS vendor to provide an integral solution for an LSDAS system.

\section{Digital Data Access and recording}

Digitized data from the LSDAS must be provided to a network interface in order for recording to occur. Due to the fact that the final selection of the LSDAS signal conditioning and digitization equipment has not been made, a multitude of options are still under consideration for the optimum method and protocol to be used for the LSDAS network interface. Emphasis will be placed upon current technology. Some level of technical maturity and stability of the technology will be required as well as open architectures and protocols such as fibre-channel, Universal Serial Bus (USB), or ethernet for this network interface.

\section{LSDAS Data Processing System}

After test, LSDAS data is converted to Engineering Unit data and transmitted to the appropriate personnel for facility or US engine analyses. Data is electronically transmitted to off-site clients after test. 


\section{Controls}

Programmable Logic Controllers (PLCs) using ethernet interconnections form the backbone of the control systems for the A-3 test facility. Their primary function is to control facility operations and sequence rocket engine tests in a safe manner. During automated testing, PLCs provide control for motor valves and variable-position valves. Several PLCs are to be used for the A-3 test facility. The control system architecture is shown in Figure 5. One PLC controls the overall facility integration and control system supervisory functions. Another PLC controls operations at the barge docks, such as transfer of propellants from storage barges to the run tanks. Another PLC will perform HSDAS and LSDAS calibration operations. Another PLC serves as the supervisory controller for the hazardous gas and fire detection system, which detects hazardous conditions and provides warning to test operations personnel. Another PLC provides primary test sequencing, communications with the test article controller and test parameter monitoring. Finally, a redundant set of PLCs is employed for operation of the Chemical Steam Generator system.

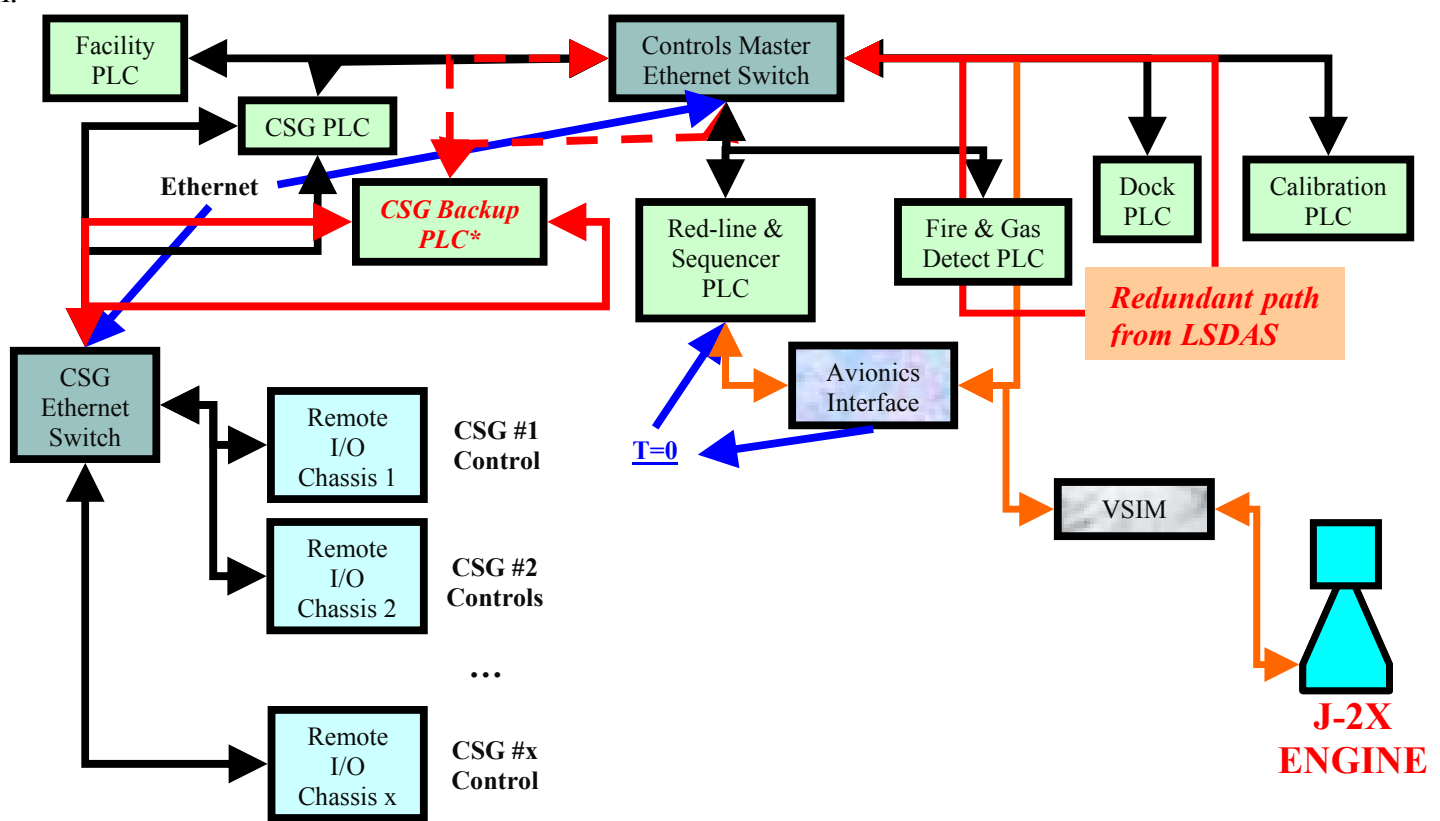

Figure 5 : Proposed A-3 Control System Architecture ${ }^{4}$

\section{Digital LSDAS Transmission to Control System}

Digitized data from the LSDAS at SSC has never been transmitted to the control systems other than for display purposes. Varying customer requirements and the inability to provide a deterministic data stream are other reasons for data not being transmitted. Therefore, for control system access to analog data, an additional signal conditioning output has historically been used to wire sensor data to the control system. This is depicted in the top portion of Figure 6.

For the A-3 test facility, an opportunity to implement advances in technology exists with respect to network data transmission. Control system processor advancements in concert with the advent of fibre-channel, reflective memory, and gigabit ethernet technology allow the MM\&CS to potentially use LSDAS outgoing data streams for deterministic use of an LSDAS data stream for control system operations. Fibre-channel is a communications bus that performs high speed data transfer. Reflective memory is a technology, in which the memory locations resident within the host system, in this case, the LSDAS digitizer, is reflected within the destination system immediately. Such a proposed implementation is depicted in the bottom Figure 6. Gigabit ethernet is a relatively new technology which is becoming widely implemented throughout many facilities. This is the next generation of the current 100 megabit ethernet technology. Gigabit ethernet is not necessarily a deterministic method of transmitting data; however, techniques may be employed to allow gigabit ethernet to be sufficiently deterministic for control system purposes. Such techniques include using "point-to-point" connections and using ethernet switches instead of hubs because switches allow programmed rules for routing of messages. To be used at the A-3 test facility, a redundant connection must be provided in case of failure of the primary connection. If gigabit ethernet is used, a "managed" 
ethernet switch should be employed which allows automatic switchover to the secondary input stream in case of failure of the primary input stream.

Due to the uncertainty of the maturity level of the technology available to accomplish LSDAS data streaming to the control system, NASA has commissioned a trade study be performed to evaluate the feasibility of implementing such a technology for the A-3 test facility ${ }^{5}$. This trade study is to consider the maturity level of the technology available, the costs associated with implementation of each option, and the schedule considerations of each option. At this point in time, this trade study is still ongoing.

\section{Typical Control System Type Inputs}

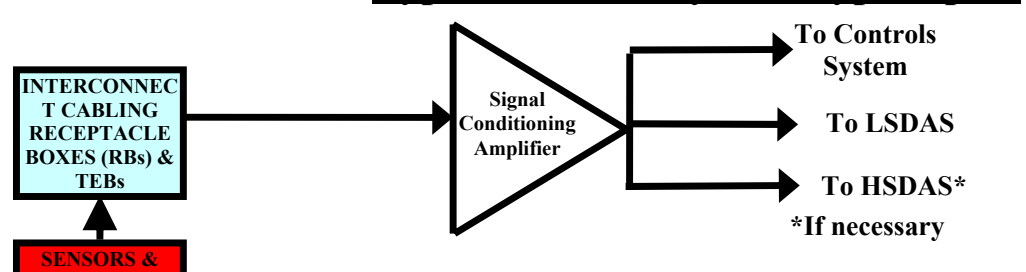

\section{Networked Control System Connection}

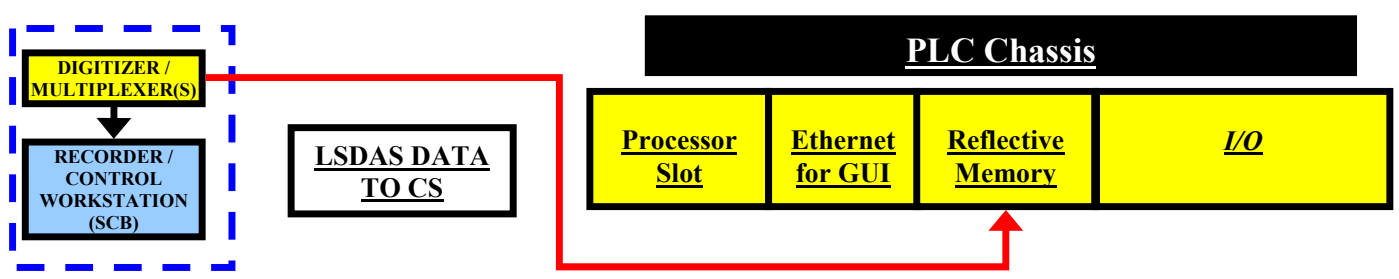

\section{Figure 6: Control system data input options ${ }^{6}$}

\section{Low and High Speed Video systems}

The Low and High Speed Video System (HSVS) to be employed at the A-3 test facility can take advantage of advances in network technology, particularly the introduction of gigabit ethernet technology, to provide high frame rate recording to digital media. To date, the video systems at SSC record to either S-VHS tape or VHS tape, depending upon the frame rates to be recorded. High Speed Video is typically recorded at 250 frames per second (fps) and Low Speed Video is typically recorded at 30 fps. The cameras must also withstand a vacuum environment of 0.08 psia, therefore, the camera housings will have to accommodate cycles from low pressure to atmospheric conditions of $14.7 \mathrm{psia}$. Camera outputs are digital connections and routed through the test cell membrane to the SCB on the ground where fiber conversion is performed for connection to the TCC. IRIG-B insertion is performed in the TCC for timing correlation across all cameras recorded as well as for comparison with the High and Low Speed DAS recording systems. A video switcher is connected to the system to allow switching from any camera to any monitor. A controller is used for camera control, such as pan, tilt, zoom, and iris control, and switching to a monitor view from any camera location. Video is recorded to digital hard drives for both High and Low Speed Video. A potential architecture for the A-3 test facility video system is depicted in Figure 7. 


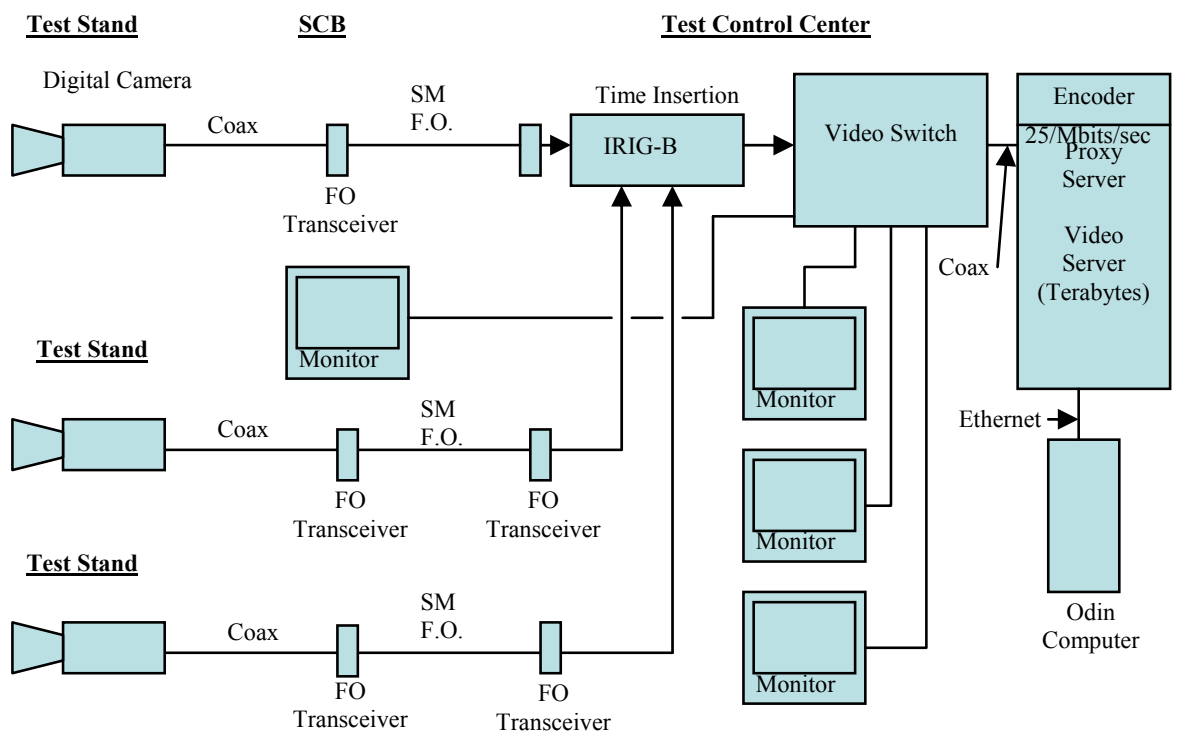

Figure 7 : Low Speed Video System for A-3 test facility ${ }^{7}$

VII. Test Facility Local Area Network (LAN)

The Test Facility Local Area Network (LAN) to be employed at the A-3 test facility will implement a gigabit ethernet network, providing interconnection between all of the A-3 Measurement, Monitor, and Control System (MM\&CS) sub-systems, as well as providing secure connections to the Stennis Data Center (SDC) and connections to stream live video and data through the SSC LAN. A gigabit ethernet switch located in the TCC is used to provide all of these connections. This switch connects to each MM\&CS sub-system LAN such as the LSDAS LAN to provide overall interconnection and intercommunications. A data transmission server with a firewall card is depicted to show outgoing live data streaming to clients outside of the test facility LAN, as well as to off-site clients. This firewall card is used to provide an outgoing only connection as well as to prevent incoming connections to the test facility LAN. A video proxy server provides similar connectivity and functionality to the Low Speed Video network. A potential architecture for the A-3 test facility overall network is depicted in Figure $8 .{ }^{8}$

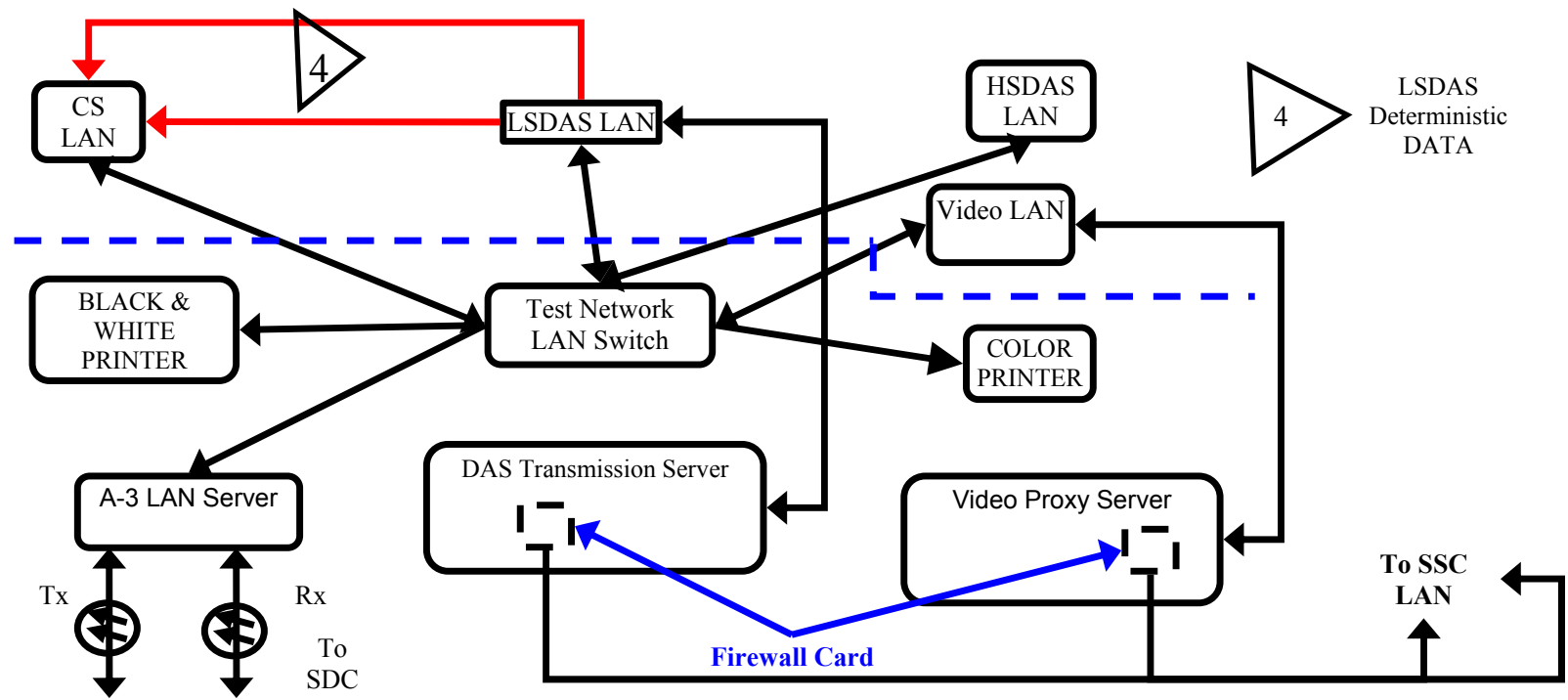

Acronyms

$\begin{array}{ll}\text { CS } & \text { Control System } \\ \text { LSDAS } & \text { Low Speed Data Acquisition System }\end{array}$

$\begin{array}{ll}\text { HSDAS } & \text { High Speed Data Acquisition System } \\ \text { DAS } & \text { Data Acquisition System }\end{array}$

$\begin{array}{ll}\text { DAS } & \text { Data Acquisition Systen } \\ \text { LAN } & \text { Local Access Network }\end{array}$

SDC Stennis Data Center

SSC Stennis Space Center

Tx Transmit

Rx Receive

TCC Test Control Center 


\title{
$\underline{\text { Figure } 8 \text { : A-3 Test Facility LAN }{ }^{9}}$
}

\section{Conclusion}

The A-3 test facility Measurement, Monitor, and Control Systems (MM\&CS) are extremely complex systems whose sole purpose is to provide accurate, reliable data to a myriad of customers in the safest possible manner. Advances in communications systems, such as the advent of gigabit ethernet and fibre-channel can allow modernizations and MM\&CS enhancements to improve automation and to integrate new technologies without compromising data integrity and reliability. With each step forward, the MM\&CS of the A-3 test facility can be utilized and upgraded to serve missions to the moon, Mars and beyond.

\section{Acknowledgments}

P. W. Hebert would like to thank his talented and knowledgeable co-workers including immediate supervisors, for assisting him in making this paper a reality. Their timely inputs, patience and technical insight were invaluable in its creation and success.

\section{References}

\author{
Presentation to the Mississippi Engineering Society (MES), March, 2008 \\ A-3 Test Stand Integrated Preliminary Design Review (I-PDR) presentation, February 2008 \\ Trade Study report for Optimum Signal Conditioning Channels per Card, George Drouant, March, 2008. \\ A-3 Test Stand Integrated Preliminary Design Review (I-PDR) presentation, February 2008 \\ Trade Study presentation for LSDAS to Control System data transmission, Joe Diggs, NTOG, December, 2007 \\ Presentation to the Mississippi Engineering Society (MES), March, 2008 \\ 200GT-GE06, Low Speed Video Specifications for the A-3 Test Stand \\ A-3 Test Stand System Architecture Document, T. Nicolaides. P. Hebert, May, 2007 \\ Presentation to the Mississippi Engineering Society (MES), March, 2008
}

